\title{
Contradictions between different measures of quantum uncertainty
}

\author{
Paloma Matía-Hernando and Alfredo Luis* \\ Departamento de Óptica, Facultad de Ciencias Físicas, Universidad Complutense, 28040 Madrid, Spain
}

(Received 29 July 2012; published 9 November 2012)

\begin{abstract}
We show that variance and Shannon entropy provide contradictory conclusions for the uncertainty associated with the number operator for some families of states of harmonic oscillator systems with fixed mean number, and for the uncertainty of a spin component for states with and without fixed mean. We analyze this behavior in terms of the properties of these uncertainty measures. We explore their impact on quantum metrology, examining the limits to resolution caused by number fluctuations in diverse scenarios of phase-shift detection.
\end{abstract}

DOI: 10.1103/PhysRevA.86.052106

PACS number(s): 03.65.Ta, 03.65.Ca, 42.50.Lc, 89.70.Cf

\section{INTRODUCTION}

Uncertainty plays a key role in quantum physics, as exemplified by basic items such as uncertainty relations. This relevance extends to practical applications such as quantum metrology, since it is believed that quantum uncertainty limits the resolution that can be achieved in any detection process [1-5].

Quantum uncertainty is usually assessed in terms of variance. Variance only properly fits with Cartesian unbounded variables with Gaussian statistics. Otherwise it may not always be a well-behaved estimator of uncertainty [6]. For example, for finite-dimensional systems, variance does not lead to meaningful uncertainty relations [7]. Moreover, for periodic variables, such as angle and phase, variance is ambiguous and rather useless because it strongly depends on the angle or phase window [8].

This has prompted the introduction of alternative measures of fluctuations [7-13]. Most of these proposals point to entropy, in particular Shannon entropy, as a suitable alternative to variance.

In this work, we compare variance- and Shannon-entropybased uncertainty measures in two different situations. First we consider the number variable, i.e., the energy, of a harmonic oscillator for some families of states with fixed mean number (Sec. II). Then we focus on the uncertainty of a spin component for states with and without fixed mean (Sec. III).

For the harmonic oscillator case, we contrast the predictions of variance and entropy, applying them first to classical states with the same mean number. We choose this arena for comparison for several reasons. Classical states make the comparison simpler since they introduce no counterintuitive nonclassical behavior. The equality of mean number is relevant for metrological applications, where resolution in the detection of weak signals depends on the mean number of particles of the probe state. Moreover, the pure-mixed comparison is relevant since it is usually understood that mixed states provide larger uncertainty than pure ones. In this regard, the only pure classical states of the harmonic oscillator are the coherent states, usually considered as the classical states with minimum uncertainty in different contexts. Finally, classical states are crucial to quantum metrology since they are easily prepared

\footnotetext{
*alluis@ fis.ucm.es; http://www.ucm.es/info/gioq
}

with very large energies and are robust against practical imperfections, such as losses and inefficient detection.

The result we obtain in this scenario is that variance and Shannon entropy provide contradictory conclusions when used as uncertainty measures. Variance predicts that mixed classical states have larger number uncertainty than pure classical states of the same mean number. Shannon entropy predicts exactly the opposite. The entropic result is rather contrary to standard intuition that points to mixed classical states as having larger uncertainty than pure ones. We also compute variance and entropy for two parametrized families including nonclassical states, and obtain equivalent results.

In Sec. III, we show that this behavior is reproduced in finite-dimensional spaces by extending the analysis to a spin component. We also remove the constraint of fixed mean value and we examine how common this anomalous feature of getting opposite variance and entropy is. To this end, we compute variance and entropy for a large number of randomly generated statistics and count the number of contradictory outcomes. This shows that this opposite behavior is not rare.

Finally, in Sec. IV, these results are contrasted in a metrology framework. It is shown that these measures of uncertainty are not conclusive concerning which states provide larger resolution when estimated using Fisher information.

Previous works have already shown that different measures of uncertainty can lead to contradictory uncertainty relations in finite-dimensional systems [13]. Moreover, it has been shown that depending on the assessment of uncertainty employed, complementary observables may satisfy no uncertainty relation [12]. It has also been shown that mixed states may provide larger resolution than pure states in nonlinear quantum metrology [5].

\section{UNCERTAINTY IN THE EXCITATION NUMBER OF A HARMONIC OSCILLATOR}

We consider a one-dimensional harmonic oscillator suitably described by the annihilation and creation operators $a, a^{\dagger}$, with $\left[a, a^{\dagger}\right]=1$. In practical terms this may be representing a one-mode electromagnetic field, a trapped ion, or a resonant $L C$ circuit.

The observable to be considered is the quantum number, represented by the operator $\hat{n}=a^{\dagger} a$, with natural-number eigenvalues $\hat{n}|n\rangle=n|n\rangle$, with $|n\rangle$ being the number states. 
The number statistics is $p_{n}=\langle n|\rho| n\rangle$, where $\rho$ is the density matrix.

\section{A. Uncertainty measures}

For uncertainty measures, we will consider variance

$$
V=\overline{n^{2}}-\bar{n}^{2}, \quad \overline{n^{k}}=\sum_{n=0}^{\infty} n^{k} p_{n},
$$

and Shannon entropy [9]

$$
S=-\sum_{n=0}^{\infty} p_{n} \ln p_{n} .
$$

Let us briefly discuss some points about these assessments. For the states considered in this work, the mean number is large and we may treat $n$ as a Cartesian-like continuous variable.

In such a case, it holds that

$$
S_{p}=-K\left(p \| p_{G}\right)+S_{p_{G}},
$$

where the subscript on $S$ indicates the number distribution. Also, $p_{G, n}$ is a Gaussian number distribution with the same mean and variance as the distribution $p_{n}$, while $K\left(p \| p_{G}\right)$ is the Kullback-Liebler divergence expressing the probability of confusing $p_{n}$ and $p_{G, n}$ under finite samplings [14],

$$
K\left(p \| p_{G}\right)=\int d n p_{n} \ln \frac{p_{n}}{p_{G, n}} .
$$

This can be regarded as a degree of non-Gaussianity [15],

$$
\mathcal{N}_{G}=K\left(p \| p_{G}\right)=S_{p_{G}}-S_{p},
$$

where $\mathcal{N}_{G} \geqslant 0$, with the equality holding if and only if $p_{n}=p_{G, n}$ for all $n$. On the other hand, for Gaussian statistics, variance and Shannon entropy are closely related,

$$
S_{p_{G}}=\ln \sqrt{2 \pi e V}
$$

Thus we may say that the information conveyed by $S_{p}$ can be split into just two contributions: number variance and nonGaussianity,

$$
S_{p}=-\mathcal{N}_{G}+\ln \sqrt{2 \pi e V} \leqslant \ln \sqrt{2 \pi e V},
$$

and we recall that by the definition of $p_{G}$, the variance $V$ is the same for the two distributions $p$ and $p_{G}$.

Shannon entropy depends only on the statistics $p_{n}$ and not on the particular values of the variable we may assign to each $p_{n}$. This is to say, the entropy for the observables $\hat{n}^{k}$ is the same for all $k$. This also holds for any $f(\hat{n})$ provided that the transformation $n \rightarrow f(n)$ is one to one. This equality no longer holds for the variance,

$$
S\left(n^{\ell}\right)=S\left(n^{k}\right), \quad V\left(n^{k}\right) \neq V\left(n^{\ell}\right),
$$

for all $k \neq \ell$.

Both $V$ and $S$ are concave functions of the statistics,

$$
\begin{aligned}
& S_{\lambda p+(1-\lambda) q} \geqslant \lambda S_{p}+(1-\lambda) S_{q}, \\
& V_{\lambda p+(1-\lambda) q} \geqslant \lambda V_{p}+(1-\lambda) V_{q},
\end{aligned}
$$

for $\lambda \in[0,1]$. More specifically, for the variance, we have

$$
V_{\lambda p+(1-\lambda) q}=\lambda V_{p}+(1-\lambda) V_{q}+\lambda(1-\lambda)\left(\bar{n}_{p}-\bar{n}_{q}\right)^{2},
$$

where $\bar{n}_{p}$ represents the mean number with respect to the distribution $p_{n}$. This leads to the common intuition that mixed states have larger fluctuations than equivalent pure states. This is clearly so if $V_{p}=V_{q}, S_{p}=S_{q}$, but might not be the case otherwise.

\section{B. Classical states with fixed mean number}

First we will focus on a family of classical states, then extend the analysis to nonclassical states in Sec. IIC. For harmonic oscillators, there is wide consensus that classical states have $s$-ordered phase-space representatives that are compatible with classical statistical physics $[16,17]$ (nevertheless, see Ref. [18]). In other words, they are non-negative and no more singular than a $\delta$ function.

\section{Pure classical state}

The only pure classical states are the coherent states $|\alpha\rangle$, which are eigenstates of the annihilation operator $a|\alpha\rangle=\alpha|\alpha\rangle$, where $\alpha$ is any complex number [19]. Any classical state is an incoherent mixture of coherent states with positive weights,

$$
\rho_{\text {class }}=\int d^{2} \alpha P(\alpha)|\alpha\rangle\langle\alpha|, \quad P(\alpha) \geqslant 0 .
$$

In the number basis $|n\rangle$, the coherent states $|\alpha\rangle$ read

$$
|\alpha\rangle=e^{-|\alpha|^{2} / 2} \sum_{n=0}^{\infty} \frac{\alpha^{n}}{\sqrt{n !}}|n\rangle, \quad \bar{n}=|\alpha|^{2} .
$$

This is a Poissonian distribution, which for large $\bar{n}$ can be well approximated by a Gaussian distribution treating $n$ as a continuous Cartesian-like variable,

$$
p_{n}=e^{-\bar{n}} \frac{\bar{n}^{n}}{n !} \simeq \frac{1}{\sqrt{2 \pi \bar{n}}} \exp \left[-\frac{(n-\bar{n})^{2}}{2 \bar{n}}\right] \text {. }
$$

The number uncertainties are

$$
V_{\text {pure }}=\bar{n}, \quad S_{\text {pure }} \simeq \ln \sqrt{2 \pi e \bar{n}},
$$

in accordance with Eq. (2.6). The Shannon entropy has been computed using the continuous number and Gaussian approximations valid for coherent states and $\bar{n} \gg 1$.

\section{Mixed classical state}

We will consider the following mixed classical state:

$$
\rho=(1-v)|0\rangle\langle 0|+v| \sqrt{\bar{n} / v}\rangle\langle\sqrt{\bar{n} / v}|,
$$

where $v$ is a real parameter between 0 and $1,|0\rangle$ is the vacuum state (which is simultaneously the number and coherent state $n=\alpha=0$ ), and $|\sqrt{\bar{n} / \nu}\rangle$ is a coherent state with $\alpha=\sqrt{\bar{n} / \nu}$. For $v=1$, the states (2.12) and (2.15) coincide. This state is specifically constructed to exploit particular features of variance at fixed mean number $\bar{n}$, especially with regard to quantum metrology [3,5]. The mean number $\bar{n}$ does not depend on $v$, so $\bar{n}$ is always the same for pure and mixed states throughout this work.

The degree of purity can be assessed by $\operatorname{tr}\left(\rho^{2}\right)$. For $\bar{n} \gg 1$, so that $\langle 0 \mid \sqrt{\bar{n} / v}\rangle \simeq 0$, the trace becomes

$$
\operatorname{tr}\left(\rho^{2}\right) \simeq 1-2 v(1-v)
$$


The maximum mixedness holds for $v=1 / 2$ and the minimum holds for $v=0,1$.

The exact number distribution of state $(2.15)$ is

$$
p_{0}=1-v+v e^{-\bar{n} / \nu}, \quad p_{n>0}=v e^{-\bar{n} / v} \frac{(\bar{n} / \nu)^{n}}{n !} .
$$

In the case $\bar{n} \gg 1$, and taking into account the continuousnumber and Gaussian approximations, the number distribution (2.17) can be approximated by

$$
p_{0} \simeq 1-v, \quad p_{n>0} \simeq v \sqrt{\frac{v}{2 \pi \bar{n}}} \exp \left[-\frac{(n-\bar{n} / v)^{2}}{2 \bar{n} / v}\right] .
$$

The corresponding number uncertainties are

$$
\begin{aligned}
& V_{\text {mix }}=\bar{n}+\frac{1-v}{v} \bar{n}^{2}, \\
& S_{\text {mix }} \simeq-(1-v) \ln (1-v)+v \ln \sqrt{2 \pi e \bar{n} / v^{3}},
\end{aligned}
$$

where the Shannon entropy has been obtained after Eq. (2.18). For $v \rightarrow 1$, both tend to the pure-state values (2.14), while for $v \rightarrow 0$, the variance diverges as $V_{\text {mix }} \simeq \bar{n}^{2} / v$, and in the same limit the entropy tends to zero as $S_{\text {mix }} \simeq v \ln \sqrt{2 \pi e \bar{n} / v^{3}}$.

In the continuous-number approximation, the number distribution for the mixed state in Eq. (2.18) is no longer Gaussian for $v \neq 1$. This departure can be assessed by the non-Gaussianity (2.5)

$$
\begin{aligned}
\mathcal{N}_{G}= & (1-v) \ln (1-v)-v \ln \sqrt{2 \pi e \bar{n} / v^{3}} \\
& +\ln \sqrt{2 \pi e\left[\bar{n}+\bar{n}^{2}(1-v) / v\right]}
\end{aligned}
$$

which naturally tends to zero as $v$ tends to one, and diverges as $\mathcal{N}_{G} \simeq \ln \sqrt{2 \pi e \bar{n}^{2} / v}$ when $v \rightarrow 0$.

\section{Variance versus Shannon entropy}

By comparing Eqs. (2.14) and (2.19), we readily get that for all $v \neq 1$, the variance is larger for the mixed state $V_{\text {mix }}>$ $V_{\text {pure }}$. Actually, as $v \rightarrow 0$, we get $V_{\text {mix }} \rightarrow \infty$. This is very general since it can be easily seen that for fixed $\bar{n}$, the classical state with minimum variance is the pure coherent state (or its phase-averaged counterparts that have the same number statistics). This is to say that, roughly speaking, for fixed $\bar{n}$, variance represents the distance to Poissonian distribution.

However, the behavior of the Shannon entropy is different. As shown in Figs. 1 and 2, the Shannon entropy of the mixed state is smaller than that of the pure state (horizontal line) unless $v$ is very close to one (throughout this work, plotted quantities are dimensionless). Additionally, when $v \rightarrow 0$, we get $S_{\operatorname{mix}} \rightarrow 0$, which is fully opposite to the behavior of variance that in the same limit diverges. The numerical evaluation of $S_{\text {mix }}$ from the exact expression and the approximation (2.19) are almost indistinguishable even for low mean numbers, and, for example, for $\bar{n}=10$, the maximum relative difference between them is $0.3 \%$. Figure 2 also reflects that after Eq. (2.7), increasing non-Gaussianity as $v \rightarrow$ 0 implies larger divergence between $S_{\text {mix }}$ and $\ln \sqrt{2 \pi e V_{\text {mix }}}$.

The maximum entropy for fixed $\bar{n}$ is provided by the exponential distribution

$$
p_{e, n}=\frac{1}{\bar{n}+1}\left(\frac{\bar{n}}{\bar{n}+1}\right)^{n},
$$

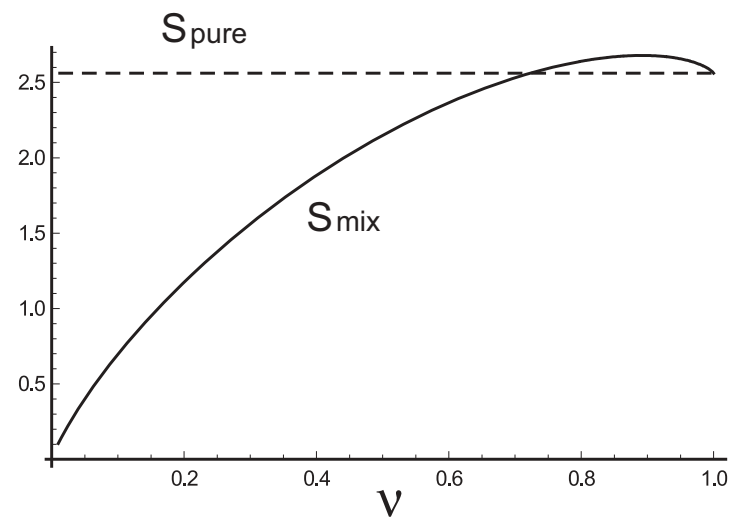

FIG. 1. Numerical evaluation of the Shannon entropy for the pure coherent state (2.12) (dashed line) and the classical mixed states (2.15) (solid line) as functions of $v$ for the same $\bar{n}=10$. Throughout this work, plotted quantities are dimensionless.

with

$$
\begin{aligned}
& V_{e}=\bar{n}(\bar{n}+1) \simeq \bar{n}^{2}, \\
& S_{e}=(\bar{n}+1) \ln (\bar{n}+1)-\bar{n} \ln \bar{n} \simeq \ln (e \bar{n}),
\end{aligned}
$$

where the approximations hold for $\bar{n} \gg 1$. We may define a measure of nonexponentiality $\mathcal{N}_{e}$ analogous to the nonGaussianity in Eq. (2.5) as

$$
\mathcal{N}_{e}=S_{p_{e}}-S_{p}=K\left(p \| p_{e}\right)=\sum_{n=0}^{\infty} p_{n} \ln \frac{p_{n}}{p_{e, n}},
$$

where $p_{e, n}$ is the exponential number distribution with the same mean number $\bar{n}$ as $p_{n}$. Therefore, for fixed $\bar{n}$, we have that $S_{p}$ is essentially the distance to the exponential distribution. Alternatively to Eq. (2.7), we may say that the information conveyed by $S_{p}$ can be split into just two contributions: mean number and nonexponentiality,

$$
S_{p} \simeq-\mathcal{N}_{e}+\ln (e \bar{n})
$$

where for simplicity we have considered $\bar{n} \gg 1$.

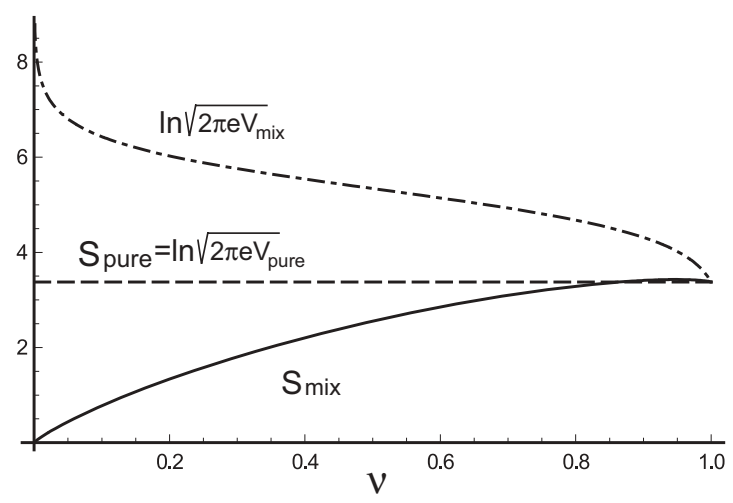

FIG. 2. Shannon entropy (solid line) and logarithm of the square root of variance (dash-dotted line) for a mixed classical state (2.15) as functions of $v$, as well as the Shannon entropy for pure coherent states (2.12) (dashed line) for $\bar{n}=50$. 


\section{Nonclassical states with fixed mean number}

We now consider the case of parametrized families of nonclassical states. For simplicity, we consider the pure-state counterpart of the family (2.15) for the same mean number $\bar{n}$ and, using the same notation, we get

$$
|\psi\rangle=\sqrt{1-v}|0\rangle+\sqrt{\nu}|\sqrt{\bar{n} / v}\rangle
$$

For $\bar{n} \gg 1$ and $v \neq 1$, this is a family of coherent superpositions of distinguishable states, also known as Schrödinger cat states [20]. Note that since $\langle 0 \mid \sqrt{\bar{n} / v}\rangle \simeq 0$, the number statistics of the families (2.15) and (2.25) are approximately the same in Eq. (2.18), so that both lead to the same results.

Another interesting family of nonclassical states with the same fixed mean number $\bar{n}$ is

$$
|\psi\rangle=\sqrt{1-v}|0\rangle+\sqrt{v}|\bar{n} / v\rangle
$$

where $|\bar{n} / \nu\rangle$ denote number states, assuming that $\bar{n} / v$ is always an integer. In this case,

$$
V=\bar{n}^{2} \frac{1-v}{v}, \quad S=-(1-v) \ln (1-v)-v \ln v .
$$

The most interesting situation for our purposes holds for $v \rightarrow$ 0 since then $V \rightarrow \infty$ while $S \rightarrow 0$, which reproduces the entropy-variance contradiction.

The main difference of this last example compared with the preceding ones is that the number statistics is nonclassical for all $v \neq 0$, in the sense that it cannot be reproduced by any classical state (2.11). We may ask whether this nonclassicality is somehow reflected by variance or entropy. Concerning number variance, nonclassical behavior is equivalent to subPoissonian statistics $\Delta n<\sqrt{\bar{n}}$, while the state (2.26) for $v \simeq 0$ is actually super-Poissonian. On the other hand, the preceding examples show that classical states can reach any value of entropy. Therefore, there seems to be no implication of nonclassicality in these results. This issue is further examined below.

\section{FINITE-DIMENSIONAL CASE}

Similar contradictory and counterintuitive results are obtained in finite-dimensional spaces, such as those associated with an angular momentum or spin system $j$ with dimension $2 j+1$. We consider dimensionless angular momentum operators $\boldsymbol{j}=\left(j_{1}, j_{2}, j_{3}\right)$ satisfying the commutation relations

$$
\left[j_{k}, j_{\ell}\right]=i \sum_{n=1}^{3} \epsilon_{k, \ell, n} j_{n}, \quad\left[j_{0}, \boldsymbol{j}\right]=\mathbf{0},
$$

where $\epsilon_{k, \ell, n}$ is the fully antisymmetric tensor with $\epsilon_{1,2,3}=1$, and $j_{0}$ is defined by the relation

$$
\boldsymbol{j}^{2}=j_{0}\left(j_{0}+1\right)
$$

The spin counterparts of the harmonic oscillator coherent states are the $\mathrm{SU}(2)$ coherent states $|j ; \theta, \phi\rangle$. In the basis $|j, m\rangle$ of eigenvectors of $j_{0}, j_{3}$, with $j_{3}|j, m\rangle=m|j, m\rangle$, they read [21]

$$
\begin{aligned}
|j ; \theta, \phi\rangle= & \sum_{m=-j}^{j}\left(\begin{array}{c}
2 j \\
m+j
\end{array}\right)^{1 / 2} \sin ^{j-m}\left(\frac{\theta}{2}\right) \\
& \times \cos ^{j+m}\left(\frac{\theta}{2}\right) e^{-i m \phi}|j, m\rangle .
\end{aligned}
$$

In this context, it is considered that classical states are the SU(2) coherent states and their incoherent combinations with positive weights $[21,22]$.

Let us consider $j_{3}$ as the observable. For definitiveness, we consider classical states with $\overline{j_{3}}=0$. Thus, the classical pure state we are going to consider is $|j ; \theta=\pi / 2, \phi\rangle$ for any $\phi$, with the following statistics for $j_{3}$ [23]:

$$
p_{j, m}=\frac{1}{4^{j}}\left(\begin{array}{c}
2 j \\
m+j
\end{array}\right) \simeq \frac{1}{\sqrt{\pi j}} e^{-m^{2} / j},
$$

where the approximation holds for large enough $j \gg 1$. The corresponding variance and entropy are

$$
V_{\text {pure }}=\frac{j}{2}, \quad S_{\text {pure }} \simeq \ln \sqrt{\pi e j} .
$$

The exact expression and the approximation (3.5) are almost indistinguishable, even for the smallest $j$. For example, for $j=1 / 2$, they differ by $5 \%$, and for $j=10$, they differ by $0.01 \%$.

For simplicity, as the mixed counterpart with $\overline{j_{3}}=0$, we consider a $50 \%$ incoherent superposition of two antipodal $\mathrm{SU}(2)$ coherent states with $\theta=0, \pi$. In the basis $|j, m\rangle$ of eigenvectors of $j_{0}, j_{3}$, this reads

$$
\rho=\frac{1}{2}(|j, j\rangle\langle j, j|+| j,-j\rangle\langle j,-j|) .
$$

The corresponding variance and Shannon entropies are

$$
V_{\text {mix }}=j^{2}, \quad S_{\text {mix }}=\ln 2,
$$

where it holds that $S_{\text {mix }}$ does not depend on $j$.

For $j>1 / 2$, here again we get that the variance is larger for the mixed state than for the pure state, $V_{\text {mix }}>V_{\text {pure }}$, in accordance with common intuition. The behavior of the Shannon entropy is different, and for $j>1 / 2$, the Shannon entropy of the mixed state is smaller than that of the pure state, $S_{\text {mix }}<S_{\text {pure }}$, as shown in Fig. 3.

\section{A. Commonness of contradictions}

All of the above analyses rely on the variance-entropy contrast on specific families of states. It is interesting to further investigate how common the variance-entropy contradictions are when they are computed for all states, removing also the constraint of fixed mean value.

To address this issue, we have performed some simple numerical calculations for the case $j=2$. Since entropy and variance only depend on the statistics of $j_{3}$, we directly generate series of five random numbers between 0 and 1 , and normalize them so that they represent suitable $j_{3}$ statistics. [This may not be the most complete approach since it does not take into account the number of states leading to the same statistics (see Ref. [24] and references therein), but we think it can provide a simple and meaningful illustration of the idea without deviating from the main issue.] 


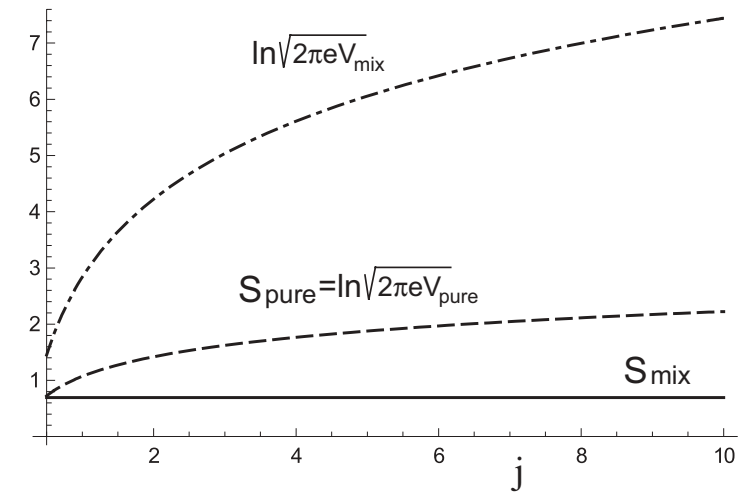

FIG. 3. Plot of the Shannon entropy (solid line) and the logarithm of the square root of the variance (dashed-dotted line) for the mixed state (3.6) as functions of $j$, as well as the Shannon entropy for the pure SU(2) coherent state (dashed line).

For each random five-tuple, we compute its variance $V$ and entropy $S$, and examine whether they are contradictory. To this end, we have defined critical values $V_{\max , \min }$ and $S_{\max , \min }$, so that $V>V_{\max }$ holds for $25 \%$ of the cases, while $V<V_{\min }$ holds for another $25 \%$ of the cases, and equivalently for entropy. Such critical values are $V_{\min }=1.49$, $V_{\max }=2.18, S_{\min }=1.37$, and $S_{\max }=1.52$. In Fig. 4, we have represented histograms for $V$ and $S$ for an ensemble of $5 \times 10^{6}$ randomly generated statistics. In Fig. 5, we have illustrated the percentages of cases in different regions of a $V-S$ plane with joint $V$ and $S$ values above and below these critical values.

Then we consider that $V$ and $S$ are contradictory when

$\left(V>V_{\max }\right.$ and $\left.S<S_{\min }\right) \quad$ or $\quad\left(V<V_{\min }\right.$ and $\left.S>S_{\max }\right)$,

which are the cases that fall within the gray squares in Fig. 5. We have found $6.5 \%$ of such contradictory cases. This is not a small figure since the number of cases with joint extreme variance and entropy,

$$
\left(V>V_{\max } \text { or } V<V_{\min }\right) \text { and }\left(S>S_{\max } \text { or } S<S_{\min }\right) \text {, }
$$

represent $23 \%$ of the total (i.e., the cases in the squares at the four corners in Fig. 5). This is to say that the number of contradictory cases is $28 \%$ of the cases that have variance and entropy large or small enough. Most of the contradictory cases (89\% of them) hold for large variance $V>V_{\max }$ and small entropy $S<S_{\min }$ (lower right square in Fig. 5).
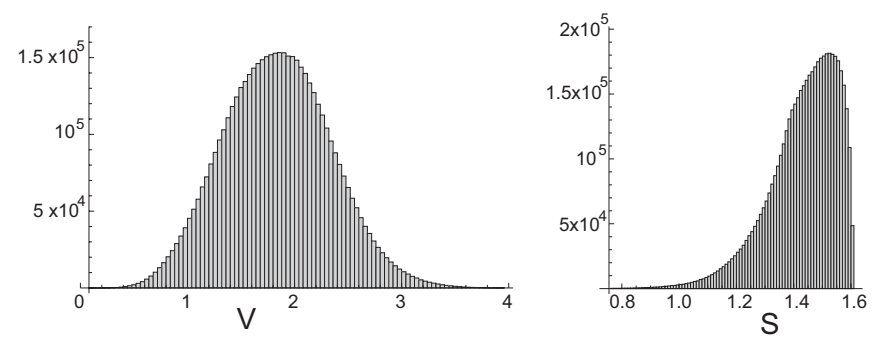

FIG. 4. Histograms for variance $V$ and entropy $S$ grouped in 100 and 200 uniformly distributed bins, respectively, for an ensemble of $5 \times 10^{6}$ randomly generated $j_{3}$ statistics.

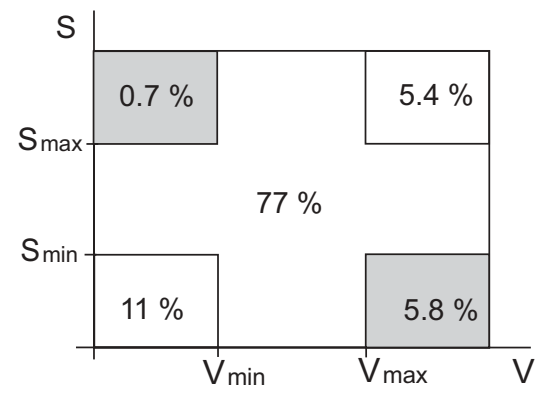

FIG. 5. Percentages of cases in different regions of a $V-S$ plane with joint $V$ and $S$ values above and below the critical values $V_{\max \text { min }}$ and $S_{\max , \min }$ in arbitrary units.

Some of these results can be simply explained. Roughly speaking, reduced variance implies that the distribution is concentrated around the mean value, so that the corresponding entropy cannot be very large. Therefore, it is natural that most examples of entropy-variance contradiction arise from large variances and small entropies (as illustrated by all of the above examples).

\section{Incidence of nonclassicality}

It may be interesting to examine whether there is any relation between nonclassicality and variance-entropy contradictions. To this end, we consider the sufficient variance-based criterion of SU(2) nonclassicality [22],

$$
\left(\Delta j_{3}\right)^{2}<\frac{j}{2}-\frac{\left\langle j_{3}\right\rangle^{2}}{2 j} .
$$

We have found that $2.8 \%$ of the total cases are nonclassical according to (3.10). $96 \%$ of them have $V<V_{\min }$ and $S<S_{\min }$ (lower left corner in Fig. 5).

In all of the runs of the numerical computation performed, we have found no single case of contradiction for a nonclassical statistics according to criterion (3.10). As we discussed above, variance-based nonclassical criteria demand small $V$ that tend to imply small $S$ as well, and therefore most nonclassical cases fall in the lower left corner in Fig. 5, excluding varianceentropy contradiction. Nevertheless, this does not exclude the possibility that there might be nonclassical statistics with contradicting entropy and variance not satisfying variancebased nonclassical criteria, as demonstrated by the example above in Sec. II C.

\section{PHASE-SHIFT RESOLUTION}

A practical framework where quantum uncertainty might play a relevant role is quantum metrology. Seemingly, the resolution in the determination of a given signal $\chi$ would be limited by quantum uncertainty [1-5].

The structure of any signal-detection scheme is quite universal. The information about some signal $\chi$ is imprinted on the state of a probe by a signal-dependent transformation $U_{\chi}=\exp (i \chi \hat{g})$ acting on a probe prepared in a known state $\rho$, where $\hat{g}$ is the generator of the transformation. The transformed state $U_{\chi} \rho U_{\chi}^{\dagger}$ is monitored by a measurement $M$ whose outputs allow one to infer the value of the signal with some uncertainty, $\Delta \chi$. 
A convenient assessment of the signal uncertainty is given by the quantum Cramér-Rao lower bound [1,25],

$$
(\Delta \chi)^{2} \geqslant \frac{1}{N F}
$$

where $N$ is the number of repetitions of the measurement, and $F$ is the quantum Fisher information,

$$
F=2 \sum_{i, j} \frac{\left(r_{i}-r_{j}\right)^{2}}{r_{i}+r_{j}}\left|\left\langle\psi_{i}|\hat{g}| \psi_{j}\right\rangle\right|^{2},
$$

where $r_{i}$ and $\left|\psi_{i}\right\rangle$ are the eigenvalues and eigenstates, respectively, of the density matrix

$$
\rho=\sum_{i} r_{i}\left|\psi_{i}\right\rangle\left\langle\psi_{i}\right|
$$

In Eq. (4.2), the sum is extended to all pairs $i, j$ with $r_{i}+r_{j} \neq$ 0 . When the system state is pure, i.e., $\rho=|\psi\rangle\langle\psi|$, we have that Eq. (4.2) greatly simplifies and becomes the variance of the generator $\hat{g}$ in the probe state $|\psi\rangle$,

$$
F=4 V(g) \text {. }
$$

In this work, we are interested in the number variable, so we consider generators proportional to the number operator, say $\hat{g}=\hat{n}^{k}$. The expression (4.4) suggests we regard $F$ as a measure of uncertainty of $\hat{g}$ (although more properly speaking, we should regard $1 / F$ as the uncertainty of the variable complementary to $\hat{g}$ ). Fisher information has already been proposed as a measure of uncertainty in Ref. [10].

Thus, Eqs. (4.2) and (4.4) provide another arena to compare the number uncertainty for the pure and mixed classical states (2.12) and (2.15), respectively. Note that in this context, the equality in mean number $\bar{n}$ for mixed and pure states is rather crucial, since it is believed that the ultimate resolution depends on the mean energy [2] (nevertheless, see Ref. [3]).

For $\bar{n} \gg 1$ so that $\langle 0 \mid \sqrt{\bar{n} / v}\rangle \simeq 0$, we have that $\rho$ would have just two eigenvectors with nonvanishing eigenvalues: $\left|\psi_{0}\right\rangle=|0\rangle$ and $\left|\psi_{1}\right\rangle=|\sqrt{\bar{n} / v}\rangle$, with eigenvalues $r_{0}=1-v$ and $r_{1}=v$, respectively. This allows one to greatly simplify the evaluation of the quantum Fisher information (4.2) in the form

$$
F=4 r_{0} V_{0}(g)+4 r_{1} V_{1}(g)-\frac{16 r_{0} r_{1}}{r_{0}+r_{1}}\left|\left\langle\psi_{1}|\hat{g}| \psi_{0}\right\rangle\right|^{2},
$$

where $V_{i}(g)$ is the variance of $\hat{g}$ in the state $\left|\psi_{i}\right\rangle$. Since $\hat{g}=\hat{n}^{k}$ and $\left|\psi_{0}\right\rangle=|0\rangle$, we have for $k \neq 0$ that $\hat{g}\left|\psi_{0}\right\rangle=0$ and $V_{0}(g)=$ 0 , so that the Fisher information simplifies even further,

$$
F=4 r_{1} V_{1}(g)=4 v V_{\alpha=\sqrt{n} / v}\left(n^{k}\right),
$$

where the subscript on $V$ indicates that this is the variance of the coherent state $|\sqrt{\bar{n} / v}\rangle$. Therefore, after Eqs. (4.4) and (4.6), the Fisher information for the pure and mixed states is

$$
F_{\text {pure }} \simeq 4 k^{2} \bar{n}^{2 k-1}, \quad F_{\text {mix }} \simeq 4 k^{2} v\left(\frac{\bar{n}}{v}\right)^{2 k-1},
$$

so that

$$
F_{\text {mix }} \simeq v^{2(1-k)} F_{\text {pure }} .
$$

For $k=1$, mixed and pure states provide the same resolution. On the other hand, for $k>1$, the mixed state provides larger resolution than the pure state, while the exact opposite holds for $k<1$. The particular case $k=2$ was presented in Ref. [5] as demonstrating the advantages of nonlinear metrology [4].

Thus, regarding Fisher information as proportional to number uncertainty, for $k>1$ the mixed state would present larger uncertainty than the pure state, and exactly the contrary for $k<1$. For exactly the number variable $k=1$, both would have the same uncertainty.

In order to connect with the preceding sections, we recall that the Shannon entropy of $n^{k}$ does not depend on $k$, i.e., $S(n)=S\left(n^{k}\right)$, while variance does depend on $k$, i.e., $V\left(n^{k}\right) \neq$ $V\left(n^{\ell}\right)$. More specifically, retaining the leading terms for $v \neq 0,1$,

$$
V_{\text {mix }}\left(n^{k}\right) \simeq v(1-v)\left(\frac{\bar{n}}{v}\right)^{2 k}, \quad V_{\text {pure }}\left(n^{k}\right) \simeq k^{2} \bar{n}^{2 k-1},
$$

we get

$$
V_{\text {mix }}\left(n^{k}\right) \simeq \frac{1-v}{v^{2 k-1} k^{2}} \bar{n} V_{\text {pure }}\left(n^{k}\right) .
$$

Thus, we have

$$
V_{\text {mix }}\left(n^{k}\right)>V_{\text {pure }}\left(n^{k}\right) \text { for } \quad \bar{n}>\frac{v^{2 k-1} k^{2}}{1-v},
$$

so that for most values of $k$ and $\bar{n}$ with practical interest (that is, $\bar{n} \gg 1$ and $k$ not very far from unity), we always have $V_{\text {mix }}\left(n^{k}\right)>V_{\text {pure }}\left(n^{k}\right)$.

Therefore, neither the variance nor the Shannon entropy reflect the key difference between $k>1$ and $k<1$ displayed by Fisher information, so we may say that they do not provide a full account of the practical consequences of uncertainty in quantum metrology.

\section{CONCLUSIONS}

We have shown that variance and Shannon entropy may provide fully contradictory conclusions when used as uncertainty measures. This suggests that all of the ramifications of uncertainty are not fully understood and that different measures of uncertainty convey different information about it. In order to analyze the reasons why these results come out the way they do, we have examined several different situations. Let us comment on the results:

(i) It could be argued that in the examples in Sec. II, holding the mean number fixed might bias the data. This is avoided by the numerical calculation in Sec. III that is carried out without any constraint, which provides a bias-free confirmation of the results.

(ii) The numerical evaluation in Sec. III shows that this phenomenon is not rare. In the particular case analyzed, the contradiction affects around $28 \%$ of the statistics that may experience it by having extreme values of variance and entropy.

(iii) The variance-entropy opposite results are both reproduced by families of classical and nonclassical states. Nevertheless, we have also shown that the statistics with contradictory outcomes tend to avoid simple variance-based nonclassical criteria.

(iv) We have examined whether the entropy-variance differences are connected with applications to quantum metrology. Focusing on the Cramér-Rao bound, we have found that 
resolution seems to be largely uncorrelated with both measures of uncertainty.

Given the relevance of uncertainty in many areas of science, we may expect implications of these results in diverse fields. Let us briefly mention some of the potential implications, future research directions, and open questions, all of them within the areas we are more familiar with:

(1) Uncertainty is naturally relevant for quantum joint uncertainty relations of noncommuting observables as well as for the emergence of complementarity. This may especially be of interest regarding the interferometric complementarity between path and visibility. This is because visibility is governed by phase relations, and phase is a typical variable where measures that are alternative to variance have already been applied [8]. Therefore, meaningful conclusions may be expected from a detailed comparison between variance and entropy. In this regard, different measures of uncertainty may lead to contradictory uncertainty relations (the same state can be either of maximum or minimum joint uncertainty), or even no uncertainty relation at all $[12,13]$.

(2) In classical optics, entropy and variance can be applied to measure the width of light beams, both in the space and angular domains. In this context, variance has already been questioned as a proper measure of beamwidth [6]. This may affect, for example, the proper characterization of the quality of a laser beam.
(3) It is worth investigating whether these variance-entropy contradictions extend to other measures of uncertainty. In particular, we may consider Tsallys and Renyi entropies that have found successful application in statistical physics [11].

(4) It might be worth examining a more complete elucidation of the relation between variance-entropy contradictions and nonclassicality.

(5) Regarding quantum metrology, we may consider two directions for possible further research. On the one hand, the Cramér-Rao bound arises from the variance of the estimator [25]. Therefore, we may investigate what happens when variance is replaced by entropy-based measures of uncertainty. On the other hand, the Cramér-Rao bound is not the only estimator of metrological resolution, and in recent times much research has been based on the Ziv-Zakai bound [26]. It might be worthwhile to investigate whether there is any correlation between variance or entropy and the Ziv-Zakai bound, and perform a parallel analysis to the one carried out in Sec. IV.

\section{ACKNOWLEDGMENTS}

A.L. acknowledges support from Project No. FIS200801267 of the Spanish Dirección General de Investigación del Ministerio de Ciencia e Innovación, and from Project No. QUITEMAD S2009-ESP-1594 of the Consejería de Educación de la Comunidad de Madrid.
[1] S. L. Braunstein, Phys. Rev. Lett. 69, 3598 (1992); S. L. Braunstein and C. M. Caves, ibid. 72, 3439 (1994).

[2] C. M. Caves, K. S. Thorne, R. W. P. Drever, V. D. Sandberg, and M. Zimmermann, Rev. Mod. Phys. 52, 341 (1980); C. M. Caves, Phys. Rev. D 23, 1693 (1981); M. J. Holland and K. Burnett, Phys. Rev. Lett. 71, 1355 (1993); M. Hillery and L. Mlodinow, Phys. Rev. A 48, 1548 (1993); J. J. Bollinger, W. M. Itano, D. J. Wineland, and D. J. Heinzen, ibid. 54, R4649 (1996); C. Brif and A. Mann, ibid. 54, 4505 (1996); Z. Y. Ou, ibid. 55, 2598 (1997); S. F. Huelga, C. Macchiavello, T. Pellizzari, A. K. Ekert, M. B. Plenio, and J. I. Cirac, Phys. Rev. Lett. 79, 3865 (1997); V. Giovannetti, S. Lloyd, and L. Maccone, Science 306, 1330 (2004); Phys. Rev. Lett. 108, 260405 (2012); M. J. W. Hall, D. W. Berry, M. Zwierz, and H. M. Wiseman, Phys. Rev. A 85, 041802(R) (2012); M. J. W. Hall and H. M. Wiseman, New J. Phys. 14, 033040 (2012); R. Nair, arXiv:1204.3761v1.

[3] A. Rivas and A. Luis, New J. Phys. 14, 093052 (2012); A. Luis and A. Rodil, arXiv:1201.3072v1; A. Luis, arXiv:1203.4325v1.

[4] A. Luis, Phys. Lett. A 329, 8 (2004); SPIE Rev. 1, 018006 (2010); M. Napolitano, M. Koschorreck, B. Dubost, N. Behbood, R. J. Sewell, and M. W. Mitchell, Nature (London) 471, 486 (2011).

[5] A. Rivas and A. Luis, Phys. Rev. Lett. 105, 010403 (2010).

[6] J. Hilgevoord, Am. J. Phys. 70, 983 (2002); G. N. Lawrence, Laser Focus World 30, 109 (1994); J. Řeháček and Z. Hradil, J. Mod. Opt. 51, 979 (2004).

[7] A. Luis, Phys. Rev. A 64, 012103 (2001); 67, 032108 (2003).

[8] J. M. Lévy-Leblond, Ann. Phys. (NY) 101, 319 (1976); E. Breitenberger, Found. Phys. 15, 353 (1985); J. B. M. Uffink, Phys. Lett. A 108, 59 (1985); J. M. Lévy-Leblond, ibid. 111, 353 (1985); S. M. Barnett and D. T. Pegg,
J. Mod. Opt. 36, 7 (1989); Z. Hradil, Phys. Rev. A 46, R2217 (1992); Quantum Opt. 4, 93 (1992); T. Opatrný, J. Phys. A 27, 7201 (1994); V. Peřinová, A. Lukš, and J. Peřina, Phase in Optics (World Scientific, Singapore, 1998).

[9] C. E. Shannon, Bell Syst. Tech. J. 27, 379 (1948); I. I. Hirschman, Am. J. Math. 79, 152 (1957); I. Bialynicki-Birula and J. Mycielski, Commun. Math. Phys. 44, 129 (1975); D. Deutsch, Phys. Rev. Lett. 50, 631 (1983); M. H. Partovi, ibid. 50, 1883 (1983); K. Kraus, Phys. Rev. D 35, 3070 (1987); H. Maassen and J. B. M. Uffink, Phys. Rev. Lett. 60, 1103 (1988); J. Sanchez, Phys. Lett. A 173, 233 (1993); Č. Brukner and A. Zeilinger, Phys. Rev. Lett. 83, 3354 (1999); Phys. Rev. A 63, 022113 (2001); M. J. W. Hall, ibid. 59, 2602 (1999); V. Majerník and E. Majerníková, Rep. Math. Phys. 47, 381 (2001); S. Massar and P. Spindel, Phys. Rev. Lett. 100, 190401 (2008); S. Wehner and A. Winter, New J. Phys. 12, 025009 (2010); I. BialynickiBirula and L. Rudnicki, Statistical Complexity, edited by K. D. Sen (Springer, Berlin, 2011), Chap. 1; A. E. Rastegin, J. Phys. A 44, 095303 (2011); Int. J. Theor. Phys. 51, 1300 (2012).

[10] M. J. W. Hall, Phys. Rev. A 62, 012107 (2000); 64, 052103 (2001); P. Sánchez-Moreno, A. R. Plastino, and J. S. Dehesa, J. Phys. A 44, 065301 (2011).

[11] A. Renyi, in Proceedings of the 4th Berkeley Symposium on Mathematics and Statistical Probability (University of California Press, Berkeley 1961), Vol. 1, pp. 547-561; C. Tsallis, J. Stat. Phys. 52, 479 (1988); U. Larsen, J. Phys. A 23, 1041 (1990); E. M. F. Curado and C. Tsallis, ibid. 24, L69 (1991); A. K. Rajagopal, Phys. Lett. A 205, 32 (1995); M. Portesi and A. Plastino, Physica A 225, 412 (1996); I. Bialynicki-Birula, Phys. Rev. A 74, 052101 (2006). 
[12] A. Luis, Opt. Lett. 31, 3644 (2006); Phys. Rev. A 75, 052115 (2007); S. Zozor, M. Portesi, and C. Vignat, Physica A 387, 4800 (2008).

[13] A. Luis, Phys. Rev. A 84, 034101 (2011).

[14] T. M. Cover and J. A. Thomas, Elements of Information Theory (Wiley Interscience, New York, 1991).

[15] P. Réfrégier and F. Goudail, J. Opt. Soc. Am. A 23, 671 (2006); J. Solomon Ivan, M. Sanjay Kumar, and R. Simon, Quantum Inf. Proc. 11, 853 (2012); arXiv:0812.2800v1.

[16] K. E. Cahill and R. J. Glauber, Phys. Rev. 177, 1857 (1969); 177, 1882 (1969); E. C. G. Sudarshan, Phys. Rev. A 23, 2802 (1981); L. Mandel and E. Wolf, Optical Coherence and Quantum Optics (Cambridge University Press, Cambridge, England, 1995); H.-W. Lee, Phys. Rep. 259, 147 (1995).

[17] C. T. Lee, Phys. Rev. A 44, R2775 (1991); 52, 3374 (1995); A. F. de Lima and B. Baseia, ibid. 54, 4589 (1996); J. Janszky, M. G. Kim, and M. S. Kim, ibid. 53, 502 (1996).

[18] J. A. Vaccaro, Opt. Commun. 113, 421 (1995); Phys. Rev. A 52, 3474 (1995); J. A. Vaccaro and D. T. Pegg, ibid. 41, 5156 (1990);
L. M. Johansen, Phys. Lett. A 329, 184 (2004); J. Opt. B 6, L21 (2004); L. M. Johansen and A. Luis, Phys. Rev. A 70, 052115 (2004); A. Luis, ibid. 73, 063806 (2006).

[19] R. L. Hudson, Rep. Math. Phys. 6, 249 (1974); M. Hillery, Phys. Lett. A 111, 409 (1985); N. Lütkenhaus and S. M. Barnett, Phys. Rev. A 51, 3340 (1995).

[20] C. C. Gerry and P. L. Knight, Am. J. Phys. 65, 964 (1997).

[21] F. T. Arecchi, E. Courtens, R. Gilmore, and H. Thomas, Phys. Rev. A 6, 2211 (1972).

[22] O. Giraud, P. Braun, and D. Braun, Phys. Rev. A 78, 042112 (2008).

[23] D. Martín and A. Luis, Phys. Rev. A 82, 033829 (2010).

[24] A. P. Majtey, A. R. Plastino, and A. Plastino, Physica A 391, 2491 (2012).

[25] H. Cramér, Mathematical Methods of Statistics (Asia Publishing House, Bombay, 1962).

[26] J. Ziv and M. Zakai, IEEE Trans. Inf. Theory 15, 386 (1969); M. Tsang, Phys. Rev. Lett. 108, 230401 (2012); V. Giovannetti and L. Maccone, ibid. 108, 210404 (2012); Y. Gao and H. Lee, J. Phys. A 45, 415306 (2012). 